\title{
Cheese-related listeriosis outbreak, Portugal, March 2009 to February 2012
}

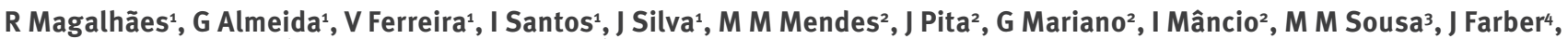

F Pagotto4, P Teixeira (pcteixeira@porto.ucp.pt) ${ }^{1}$

1. Centro de Biotecnologia e Química Fina - Laboratório Associado, Escola Superior de Biotecnologia, Universidade Católica Portuguesa/Porto, Porto, Portugal

2. Autoridade de Segurança Alimentar e Económica, Lisbon, Portugal

3. Administração Regional de Saúde de Lisboa e Vale do Tejo, Lisbon, Portugal

4. Listeriosis Reference Centre for Canada, Bureau of Microbial Hazards, Health Canada, Ottawa, Ontario, Canada

Citation style for this article:

Magalhães R, Almeida G, Ferreira V, Santos I, Silva J, Mendes MM, Pita J, Mariano G, Mâncio I, Sousa MM, Farber J, Pagotto F, Teixeira P. Cheese-related listeriosis outbreak, Portugal, March 2009 to February 2012. Euro Surveill. 2015;20(17):pii=21104. Available online: http://www.eurosurveillance.org/ViewArticle.

aspx?Articleld $=21104$

In Portugal, listeriosis has been notifiable since April 2014, but there is no active surveillance programme for the disease. A retrospective study involving 25 national hospitals led to the detection of an outbreak that occurred between March 2009 and February 2012. The amount of time between the start of the outbreak and its detection was 16 months. Of the 30 cases of listeriosis reported, 27 were in the Lisbon and Vale do Tejo region. Two cases were maternal/neonatal infections and one resulted in fetal loss. The mean age of the non-maternal/neonatal cases was 59 years (standard deviation: 17); 13 cases were more than 65 yearsold. The case fatality rate was $36.7 \%$. All cases were caused by molecular serogroup IVb isolates indistinguishable by pulsed-field gel electrophoresis and ribotype profiles. Collaborative investigations with the national health and food safety authorities identified cheese as the probable source of infection, traced to a processing plant. The magnitude of this outbreak, the first reported food-borne listeriosis outbreak in Portugal, highlights the importance of having an effective listeriosis surveillance system in place for early detection and resolution of outbreaks, as well as the need for a process for the prompt submission of Listeria monocytogenes isolates for routine laboratory typing.

\section{Introduction}

Listeria monocytogenes is an intracellular bacterial pathogen of humans and a variety of animal species. In humans, L. monocytogenes infections are mainly foodborne and can cause an invasive and often fatal disease in pregnant women and their fetuses, newborns, elderly people and immunocompromised individuals, with a case fatality rate of up to $30 \%$ [1]. The incidence of listeriosis increased in several European countries between 2009 and 2013 (such as Germany, the Netherlands, Spain and the United Kingdom [1,2]) and, was the most frequent cause of hospitalisation and death $(15.6 \%)$ due to the consumption of contaminated food in Europe in 2013 [2]. This increase reinforces the need for each country to establish enhanced molecular surveillance of listeriosis for efficient outbreak detection, investigation and control, as carried out by PulseNet USA or the Centre National de Référence des Listeria, Institut Pasteur, Paris, for example [3,4]. A similar programme for listeriosis surveillance at European Union level by harmonising methodological variables such as case definition, laboratory procedures and reporting systems is crucial. A pilot project was conducted by the European Centre for Disease Prevention and Control (ECDC) between January and March 2013 aiming to evaluate a Listeria external quality assurance scheme for the typing of $L$. monocytogenes that covered pulsed-field gel electrophoresis (PFGE) method and serological typing (both as a phenotypic and a multiplex polymerase chain reaction (PCR)-based method) [5]. Results demonstrated that the majority (59\%) of the participating laboratories were able to produce a PFGE gel of sufficiently high quality and the average score for serotyping among the participants was $94 \%$ and $97 \%$ for traditional and multiplex PCR based methods, respectively; however, higher quality could be achieved through trouble-shooting assistance and training.

In the absence of an active surveillance system for listeriosis at a national level, a collaborative study between the Listeria Research Centre of Escola Superior de Biotecnologia (LRCESB) and 25 of the major national hospitals (on a voluntary basis), covering about $90 \%$ of the population, was established in 2003 with the aim of obtaining epidemiological data on human listeriosis cases in Portugal and characterising clinical isolates of L. monocytogenes both phenotypically and genetically. In 2003, the incidence of listeriosis was 0.14 cases per 100,000 population [6]. An increase was reported between 2003 and 2007, i.e. it was 0.23 cases per 100,000 inhabitants for the year 2007 [7]. As a result of this study, an increase in the number of listeriosis cases was detected between January and July 2010, particularly in the Lisbon and Vale do Tejo region 
that corresponds to $13 \%$ of the total area of mainland Portugal and $34 \%$ of the total population (3.6 million inhabitants) [8], representing the first detected outbreak of listeriosis in Portugal. Here we describe the outbreak, as well as give details of the investigations carried out in order to determine the source of infection.

\section{Methods}

\section{Case definition}

A listeriosis case was defined as a non-maternal/ neonatal (non-MN) patient who met the laboratory criteria or a mother with a laboratory-confirmed listeriosis infection in her fetus, stillborn or newborn, as described in the Commission Decision of 28/IV/2008 [9]. Cases (laboratory confirmed with unknown clinical criteria) were detected through voluntary reporting by hospitals to the LRCESB in Porto.

If the pathogen was isolated from a pregnant woman and her newborn, stillborn or fetus, this was counted as a single case. Information regarding the sex and age of the patient, underlying pathology (if present), the tissue or fluid from which the bacteria were isolated and the year of isolation was reported.

\section{Culture collection}

Hospitals sent isolates of $L$. monocytogenes to LRCESB for species confirmation and typing. Species confirmation was performed by carbohydrate fermentation (rhamnose, xylose and mannitol) and Christie Atkins Munch-Petersen (CAMP) test [10]. Confirmed isolates of $L$. monocytogenes were stored in tryptic soy broth with $30 \%(\mathrm{v} / \mathrm{v})$ glycerol at $-80^{\circ} \mathrm{C}$ in the culture collection of the LRCESB.

\section{Molecular-serotyping}

Molecular serotype of L. monocytogenes isolates was determined by multiplex PCR according to Doumith et al. [11]. This assay differentiates five major subtypes, each representing more than one serotype: geno-serogroup IVb (serotypes $4 \mathrm{~b}, 4 \mathrm{~d}$ and $4 \mathrm{e}$ ), geno-serogroup Ila (serotypes $1 / 2 a$ and $3 a$ ), geno-serogroup IIb (serotypes $1 / 2 b, 3 b$ and 7 ), geno-serogroup IIc (serotypes $1 / 2 \mathrm{C}$ and $3 \mathrm{C}$ ) and geno-serogroup IVa (serotypes $4 \mathrm{a}$ and $4 \mathrm{c})$.

\section{Pulsed-field gel electrophoresis}

PFGE typing was performed according to the standard CDC PulseNet protocol [12] using the restriction enzymes $A s C l$ and $A p a l$ and gel run in CHEF III DR System (Bio-Rad, Laboratories, Hercules, CA, United States). Salmonella enterica serovar Braenderup H9812 (ATCC) DNA digested with $\mathrm{Xbal}$ was used as a reference size standard. Cluster analysis of the PFGE types was performed with the GelCompar software (Applied Maths, Sint-Martens-Latem, Belgium) by the unweighted pair group method with average linkages (UPGMA), using the Dice coefficient, and visually validated.
Ribotyping

Automated ribotyping was performed using the restriction enzyme EcoRI and the RiboPrinter microbial characterisation system (Qualicon Inc., Wilmington, DE, United States), as previously described $[13,14]$.

\section{Outbreak investigation}

The outbreak was investigated by the national health (Direção Geral de Saúde and Administração Regional de Saúde de Lisboa e Vale do Tejo) and food safety (Autoridade de Segurança Alimentar e Económica) authorities in collaboration with LRCESB.

A standardised questionnaire (adapted from a Canadian listeriosis outbreak, kindly supplied by Dr Jeff Farber of the Public Health Agency of Canada) was administered by the national health authority to patients diagnosed with listeriosis or their families (face-to-face interview) concerning their diet histories in the two months before symptom onset, with reference to the type of food consumed and household shopping patterns.

\section{FIGURE 1}

Distribution of human listeriosis cases in Portugal with the outbreak Listeria monocytogenes strain, March 2009_ February $2012(\mathrm{n}=30)$

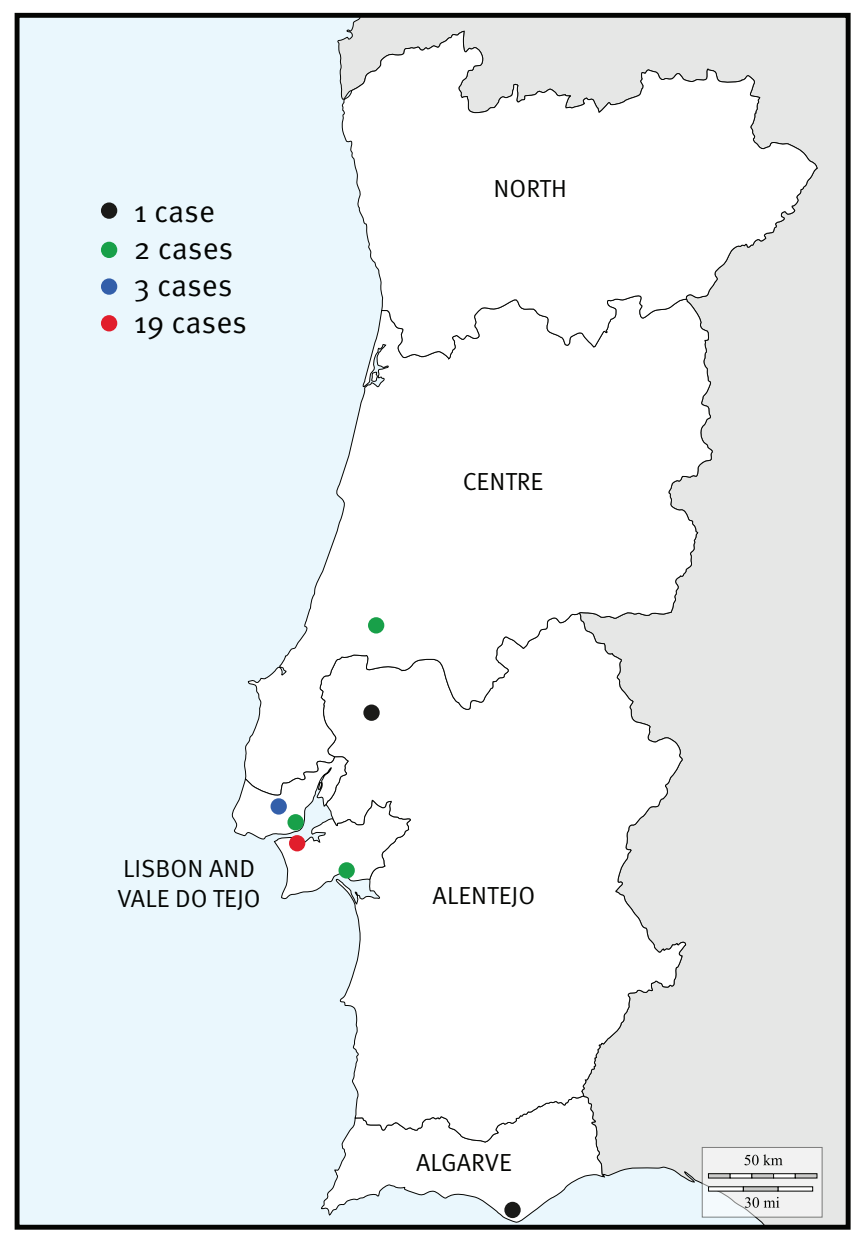

Map adapted from www.d-maps.com/m/europa/portugal/ portugal19.pdf. 


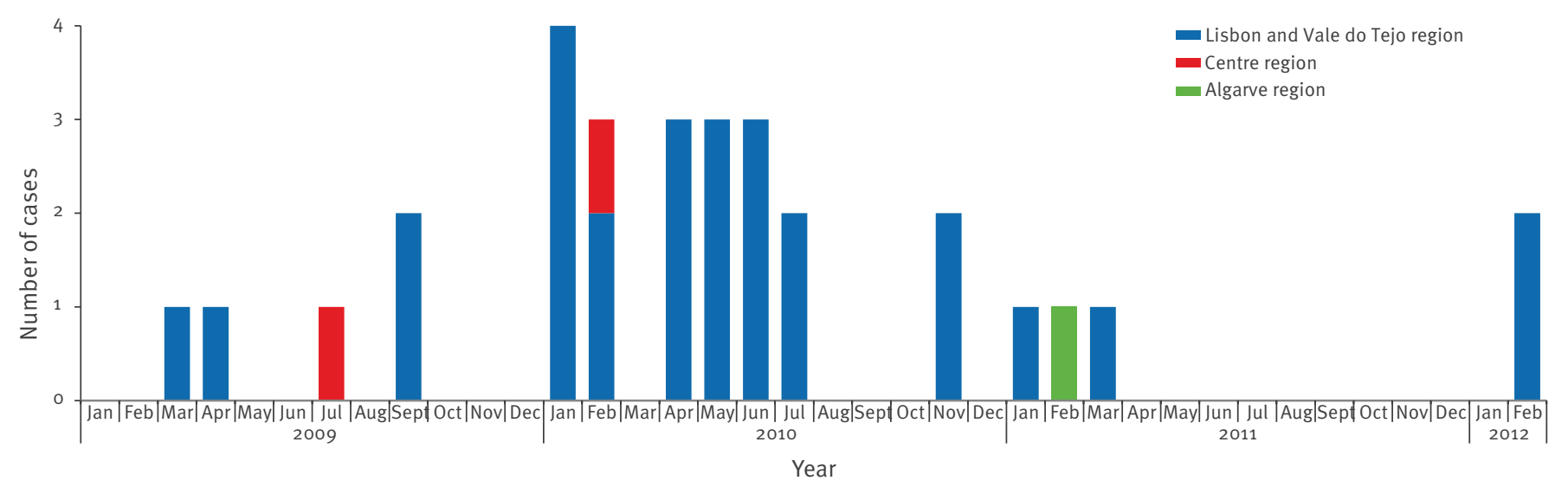

All except three cases were in the Lisbon and Vale do Tejo region (two were in Centre region, one case occurred in the Algarve region). Information on the number of listeriosis cases caused by Listeria monocytogenes strains with non-outbreak pulsed-field gel electrophoresis types for each year is available from Magalhães et al. [15].

Analysis of food products and environmental samples was conducted by the food safety authority. L. monocytogenes isolates from food and environmental samples were sent to LRCESB for typing.

\section{International enquiry}

To determine if the outbreak-associated strain of $L$. monocytogenes had been recovered from clinical or food samples from other countries, the PFGE type was communicated and compared with those of L. monocytogenes isolates in databases in France (Centre National de Référence des Listeria, Institut Pasteur), Canada (Listeriosis Reference Centre, Health Canada) and United States (Food Microbe Tracker, Food Safety Laboratory, Cornell University).

\section{Results}

\section{Recognition of the outbreak}

Between January and July 2010, a high number of listeriosis cases was observed (40 cases compared with 20 cases observed during all of 2009) [15], particularly in the Lisbon and Vale do Tejo region, where the majority of the cases were reported. Molecular typing of the $40 \mathrm{~L}$. monocytogenes clinical isolates revealed that 18 serotype IVb isolates presented the same PFGE type and ribotype, which had been observed for five isolates recovered in 2009, four of which were in the Lisbon and Vale do Tejo region (in March, April and September) and one in the Centre region (in July) (Figures 1 and 2). This PFGE type was not found in the databases searched.

In July 2010, the national health and food safety authorities were alerted to the increased number of cases and an outbreak investigation was initiated. A public health alert was issue to national hospitals requesting prompt notification and reporting of cases.
LRCESB continued to receive clinical isolates for typing. Continued monitoring detected two more cases with the outbreak strain in November 2010, and three more cases in January, February and March 2011 (two in the Lisbon and Vale do Tejo region and one in the Algarve). Thereafter, in February 2012, there were two new cases with the same strain in the Lisbon and Vale do Tejo region. The total number of outbreak cases between March 2009 and February 2012 was 30.

Trace-back and investigation of the food source Analysis of the epidemiological questionnaires pointed to different types and sizes of food retailers and identified the following as possible sources of infection: cheeses (cured cheese and queijo fresco, made from pasteurised cow and goat milk), ice cream, ham and fermented sausages. On the basis of data gathered concerning the type of establishments where the food products were purchased, as well as the geographical location of the cases, suspected foods and foods commonly associated with listeriosis, the food safety authority inspected 42 food retailers and collected 103 samples for analysis ( 51 meat products, 24 dairy products, 13 ready-to-eat foods and 15 environmental swabs). L. monocytogenes was detected in four samples collected at a retailer: three from queijo fresco and one from a swab taken from a ham slicing-machine; one queijo fresco sample contained counts of $L$. monocytogenes greater than 100 colony-forming units/g.

PFGE typing revealed that isolates recovered from two queijo fresco samples of different brands from the same retailer showed the same PFGE type as the clinical isolates with the outbreak strain. Further investigation of the processing plants where these cheeses had been produced involved collecting and testing environmental and cheese samples. The outbreak strain was 
Apal

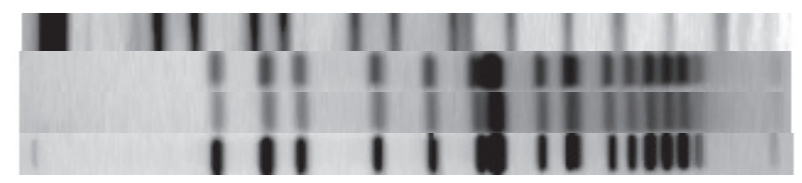

Ascl

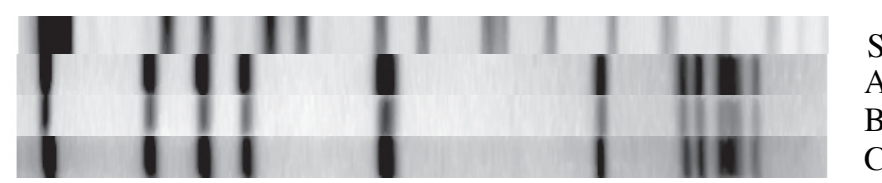

A: L. monocytogenes isolate from a listeriosis case from a hospital in the Lisbon and Vale do Tejo region (February 2010); B: L. monocytogenes isolate from a cheese sample collected at a retailer selected on the basis of the results of the epidemiological questionnaires (October 2010), C: L. monocytogenes isolate from a contaminated cheese sample collected at a processing plant located in the Alentejo region and identified by trace-back investigations (March 2011); S: Salmonella Braenderup size control.

detected in L. monocytogenes isolates from cheese samples from one of the two processing plants investigated (located in the Alentejo region) (Figure 3). Thus, cheeses produced by this plant were considered the probable source of the outbreak; cross-contamination between products or contamination from the environment, or both, may have occurred at retail level, as both suspected brands of queijo fresco were sold in the same market. As a result of these findings, the food safety authority recalled both products and more samples from the processing plant were analysed. Cheeses made with pasteurised cow and goat milk collected at the processing plant tested positive for L. monocytogenes and the collected isolates had the same PFGE pattern as the outbreak strain. Subsequently, in March 2011 the processing plant voluntarily suspended its activities during 15 days. After appropriate cleaning and disinfection measures, intensified product and environmental sampling was carried out. No positive samples were detected and products were allowed to be sold in the marketplace. Samples were then collected monthly by the food safety authority and no further positive samples have been detected.

\section{Characteristics of listeriosis outbreak- associated cases}

Of the 30 cases, two were MN cases, both of which occurred in 2010 (Table).

One $M N$ case resulted in stillbirth and the other $M N$ case involved a newborn with unknown outcome. For the 28 non-MN cases, isolates were collected from blood $(n=16)$, cerebrospinal fluid $(n=10)$ and from both blood and cerebrospinal fluid $(n=2)$. The mean age of the 27 non-MN cases with a reported age was 58.9 years (standard deviation: 17); the median was 64 years (range: 15-83); 13 non-MN cases were older than 65 years. The ratio of male:female non-MN cases was 22:6. Information was available for 20 non-MN patients with underlying conditions (e.g. diabetes mellitus, cancer, hepatitis, human immunodeficiency syndrome (HIV) infection/acquired immunodeficiency syndrome
(AIDS)). For seven non-MN cases, no such information was available. The absence of known predisposing condition was reported for one 15 year-old patient. The overall case fatality rate, for $\mathrm{MN}$ and non-MN cases, was $37 \%(11 / 30)$.

\section{Discussion}

As there is no active surveillance programme for listeriosis in Portugal, outbreak detection is extremely difficult. The incubation period of the infection can be very long, up to 70 days, which makes it difficult to find a link between cases [1]. Detection of the outbreak

\section{TABLE}

Listeriosis outbreak-associated cases, Portugal, March 2009-February $2012(\mathrm{n}=30)$

\begin{tabular}{|l|c|c|c|c|c|}
\hline \multirow{2}{*}{ Data } & \multicolumn{5}{|c|}{ Number of cases } \\
\cline { 2 - 6 } & 2009 & 2010 & 2011 & 2012 & Totals \\
\hline Clinical form & 5 & 18 & 3 & 2 & 28 \\
\hline Non-MN & 0 & 2 & 0 & 0 & 2 \\
\hline MN & 2 & 5 & 1 & 0 & 8 \\
\hline Sex & 3 & 15 & 2 & 2 & 22 \\
\hline Female & 3 & 8 & 2 & 1 & 14 \\
\hline Male & 2 & 10 & 1 & 0 & 13 \\
\hline Age of non-MN cases (years) & 0 & 0 & 0 & 1 & 1 \\
\hline$<65$ & 4 & 10 & 1 & 1 & 16 \\
\hline$\geq 65$ & 1 & 6 & 2 & 1 & 10 \\
\hline Age unknown & 0 & 2 & 0 & 0 & 2 \\
\hline Clinical sample of non-MN cases \\
\hline Blood & 3 & 7 & NA & NA & 10 \\
\hline CSF & NA & 1 & NA & NA & 1 \\
\hline Blood and CSF &
\end{tabular}

CSF: cerebrospinal fluid; MN: maternal/neonatal cases; NA: not available; non-MN: non-maternal/neonatal cases. 
reported here was due mainly to retrospective investigations. The amount of time between the presumed onset of the outbreak (March 2009) and its recognition was extremely long (16 months). This long delay amplified the magnitude of the outbreak, leading to a high number of cases $(n=30)$ and a high case-fatality rate $(36.7 \%)$. Underestimation of the number of cases is likely, as many cases usually go unreported and unrecognised, since patient data and strains are voluntarily reported and listeriosis is an infrequent disease [2], with clinical symptoms that are difficult to identify [16].

Typing of clinical and food isolates of $L$. monocytogenes by molecular techniques, such as PFGE, was essential for the identification of cheese of a specific brand as being the most probable source of contamination. Although a cheese from another producer was contaminated at retail by a strain with the outbreak-associated PFGE type, this was probably a result of cross-contamination since no positive samples were detected in the processing plant. Increased risk of cross-contamination of ready-to-eat foods by L. monocytogenes in a retail environment has been demonstrated in several studies [17-20]. For example, a quantitative risk assessment conducted by Endrikat et al. suggested that ready-toeat deli meats sliced at a retailer are five times more likely to cause listeriosis than pre-packaged products (per annum basis) [21].

Additional information is needed for a better understanding of the risk factors and for the development of improved strategies for controlling L. monocytogenes in these environments.

The long duration of this outbreak (March 2009 to February 2012) is noteworthy and reinforces the importance of setting up an effective multidisciplinary team able to help ensure rapid notification of cases and the prompt submission of L. monocytogenes isolates for routine laboratory typing.

Of the 28 non-MN cases, 13 were 65 years of age or older and at least 20 cases presented an underlying condition. In European countries with established surveillance programmes, such as France, Germany and the United Kingdom, the incidence of listeriosis is reported to be increasing and the distribution of cases is shifting, primarily affecting elderly persons and those with predisposing medical conditions, leading to a high case fatality rate $[1,2]$. This is of concern as life expectancy increases, including for those who are immunocompromised (e.g. those with AIDS, under immunosuppressive therapy for cancer) [22]. In addition, food habits are changing worldwide, with an increasing demand for processed ready-to-eat foods [23]. Therefore, it is likely that there will be an increased risk of food-borne listeriosis.

Data gathered from the surveillance of human disease and also from all stages in the food production chain should be continuously collected and analysed to understand the ecology of $L$. monocytogenes and its routes of transmission. This will be crucial for developing enhanced strategies to control this organism and contribute to a decrease in the incidence of food-borne listeriosis in Portugal.

\section{Acknowledgments}

This work was supported by funding from the National Funds from the Fundação para a Ciência e a Tecnologia (FCT) through project Pest-OE/EQB/LAo016/2011 and thorough project KLEAR - Knowledge and tools for safety, quality and competitiveness in the food chain (no. NORTE-07-0124FEDER-000052) financed by the North Portugal Regional Operational Programme (ON.2 - O Novo Norte), under the National Strategic Reference Framework (NSRF), through the European Regional Development Fund (ERDF), as well as by National Funds through the FCT. Financial support for authors Rui Magalhães and Vânia Ferreira was provided by FCT through doctoral fellowship SFRH/BD/71704/2010 and postdoctoral fellowship SFRH/BPD/72617/2010, respectively.

We kindly acknowledge the following national hospitals for prompting reporting of listeriosis cases and clinical data: Centro Hospitalar do Baixo Vouga, Entidade Pública Empresarial (EPE; Sónia Ferreira, Raquel Diaz, Elmano Ramalheira), Centro Hospitalar do Barlavento Algarvio, EPE; Centro Hospitalar Lisboa Central, EPE (Cristina Marcelo, João Marques, Isabel Daniel, Isabel Peres, Margarida F. Pinto, Odete Chantre); Centro Hospitalar Lisboa Norte, EPE (J. Melo Cristino); Centro Hospitalar de Lisboa Ocidental, EPE (Judite Batista, Teresa Marques); Centro Hospitalar do Porto (Helena Ramos, Paulo Pereira); Centro Hospitalar de Setúbal, EPE (Jesuina Duarte, Luisa Espadinha, Andrea Bernardo, Inês Tapadinhas); Centro Hospitalar do Tâmega e Sousa, EPE (Isaura Terra, Flávio Assunção, Mariana Viana); Centro Hospitalar e Universitário de Coimbra, EPE (Henrique Manuel Madeira Resende Oliveira, Luísa Boaventura); Centro Hospitalar de Vila Nova de Gaia/Espinho, EPE (Paulo Lopes, Vânia Soares); Hospital Beatriz Ângelo \& Hospital de Cascais Dr. José de Almeida (Laura Brum); Hospital de Braga (Maria Alberta Faustino); Hospital Distrital de Santarém, EPE (Paula Pinto); Hospital Dr. Nélio Mendonça, Madeira (Teresa Afonso); Hospital Espírito Santo, EPE (Adriana Coutinho); Hospital de Faro, EPE; Hospital Garcia da Horta (José Diogo, Ana Rodrigues, Isabel Nascimento); Hospital Militar de Belém (João Lago); Hospital Pedro Hispano (Maria Antónia Read); Hospital Prof. Doutor Fernando Fonseca (Luisa Sancho, Teresa Sardinha); Hospital de São João, EPE (Tiago Guimarães, Maria Dolores Pinheiro, Manuela Ribeiro); Serviço de Patologia Clínica Hospital CUF Descobertas \& CML Dr Germano de Sousa (M. Favila Menezes, M. J. Sousa, J. Germano de Sousa); Serviço de Patologia Clínica (Laboratório de Microbiologia), Hospital de Curry Cabral (Helena Peres, Teresa Pina).

The authors would also like to acknowledge Professor Martin Wiedmann from Cornell University, NY, United States, and Dr Alexandre Leclercq from the WHO Collaborating Centre for Listeria, Institut Pasteur, Paris, France, for comparing the pulsotype of the outbreak strain with those deposited in international databases. Advice on outbreak investigation by Professor Martin Wiedmann is also gratefully acknowledged.

Conflict of interest

None declared. 


\section{Authors' contributions}

Maria Manuela Sousa was responsible for the collection of epidemiological data and for the link between hospitals and Centro de Biotecnologia e Química Fina (CBQF). Autoridade de Segurança Alimentar e Económica (ASAE) team was in charge of collection and analysis of food products and environmental samples. CBQF team was responsible for collecting clinical isolates supplied by the hospitals and food isolates supplied by ASAE. CBQF and Listeriosis Reference Centre for Canada teams were responsible for characterisation of the isolates. All authors participated in the analysis and interpretation of data. Rui Magalhães, Gonçalo Almeida, Vânia Ferreira and Paula Teixeira drafted the manuscript. Jeffrey Farber and Franco Pagotto critically reviewed the draft manuscript and provided substantive input. All authors approved the final version.

\section{References}

1. Allerberger F, Wagner M. Listeriosis: a resurgent foodborne infection. Clin Microbiol Infect. 2010;16(1):16-23. http://dx.doi. org/10.1111/j.1469-0691.2009.03109.x PMID:20002687

2. European Food Safety Authority (EFSA) and European Centre for Disease Prevention and Control (ECDC). The European Union summary report on trends and sources of zoonoses, zoonotic agents and food-borne outbreaks in 2013. EFSA Journal. 2015;13(1):3991. doi:10.2903/i.efsa.2015.3991

3. Gerner-Smidt P, Hise K, Kincaid J, Hunter S, Rolando S, HyytiäTrees E, et al. PulseNet USA: a five-year update. Foodborne Pathog Dis. 2006;3(1):9-19. http://dx.doi.org/10.1089/ fpd.2006.3.9 PMID:16602975

4. Goulet V, Jacquet C, Martin P, Vaillant V, Laurent E, de Valk H. Surveillance of human listeriosis in France, 2001-2003. Euro Surveill. 2006;11(6):79-81. PMID:16801697

5. European Centre for Disease Prevention and Control (ECDC). External quality assurance scheme for Listeria monocytogenes typing. Stockholm: ECDC; 2014. Available from: http:// ecdc.europa.eu/en/publications/Publications/EQA-listeriamonocytogenes.pdf

6. Almeida GN, Gibbs PA, Hogg TA, Teixeira PC. Listeriosis in Portugal: an existing but under reported infection. BMC Infect Dis. 2006;6(1):153-6. http://dx.doi.org/10.1186/1471-2334-6153 PMID:17054782

7. Almeida G, Morvan A, Magalhães R, Santos I, Hogg T, Leclercq $A$, et al.; Research Team. Distribution and characterization of Listeria monocytogenes clinical isolates in Portugal, 19942007. Eur J Clin Microbiol Infect Dis. 2010;29(10):1219-27. http://dx.doi.org/10.1007/s10096-010-0988-x PMID:20563829

8. Instituto Nacional de Estatística (Statistics Portugal). Lisbon: Statistics Portugal. [Accessed 6 Jan 2015]. Available from: http://www.ine.pt

9. European Commission. Commission Decision of $28 / \mathrm{IV} / 2008$ amending Decision 2002/253/EC laying down case definitions for reporting communicable diseases to the Community network under Decision No 2119/98/EC of the European Parliament and of the Council. Luxembourg: Publications Office of the European Union; 2008. Available from: http://ec.europa. eu/health/ph_threats/com/docs/1589_2008_en.pdf

10. McKellar RC. Use of the CAMP test for identification of Listeria monocytogenes. Appl Environ Microbiol. 1994;60(12):4219-25. PMID:7811061

11. Doumith M, Buchrieser C, Glaser P, Jacquet C, Martin P. Differentiation of the major Listeria monocytogenes serovars by multiplex PCR. J Clin Microbiol. 2004;42(8):3819-22. http:// dx.doi.org/10.1128/JCM.42.8.3819-3822.2004 PMID:15297538

12. Graves LM, Swaminathan B. PulseNet standardized protocol for subtyping Listeria monocytogenes by macrorestriction and pulsed-field gel electrophoresis. Int J Food Microbiol. 2001;65(1-2):55-62. http://dx.doi.org/10.1016/S01681605(00)00501-8 PMID:11322701

13. Allerberger F, Fritschel SJ. Use of automated ribotyping of Austrian Listeria monocytogenes isolates to support epidemiological typing. J Microbiol Methods. 1999;35(3):23744. http://dx.doi.org/10.1016/S0167-7012(99)00025-1 PMID:10333075

14. Bruce JL, Hubner RJ, Cole EM, McDowell Cl, Webster JA. Sets of EcoRl fragments containing ribosomal RNA sequences are conserved among different strains of Listeria monocytogenes. Proc Natl Acad Sci USA. 1995;92(11):5229-33. http://dx.doi. org/10.1073/pnas.92.11.5229 PMID:7539145
15. Magalhães R, Ferreira V, Santos I, Almeida G, Teixeira P; Research Team. Genetic and phenotypic characterization of Listeria monocytogenes from human clinical cases that occurred in Portugal between 2008 and 2012. Foodborne Pathog Dis. 2014;11(11):907-16. http://dx.doi.org/10.1089/ fpd.2014.1806 PMID:25361468

16. Scallan E, Hoekstra RM, Angulo FJ, Tauxe RV, Widdowson MA, Roy SL, et al. Foodborne illness acquired in the United States -major pathogens. Emerg Infect Dis. 2011;17(1):7-15. http:/l dx.doi.org/10.3201/eid1701.P11101 PMID:21192848

17. Gombas DE, Chen Y, Clavero RS, Scott VN. Survey of Listeria monocytogenes in ready-to-eat foods. J Food Prot. 2003;66(4):559-69. PMID:12696677

18. Sauders BD, Sanchez MD, Rice DH, Corby J, Stich S, Fortes $\mathrm{ED}$, et al. Prevalence and molecular diversity of Listeria monocytogenes in retail establishments. J Food Prot. 2009;72(11):2337-49. PMID:19903398

19. Hoelzer K, Oliver HF, Kohl LR, Hollingsworth J, Wells MT, Wiedmann M. Structured expert elicitation about Listeria monocytogenes cross-contamination in the environment of retail deli operations in the United States. Risk Anal. 2012;32(7):1139-56. http://dx.doi.org/10.1111/j.15396924.2011.01729.x PMID:22053817

20. Hoelzer K, Sauders BD, Sanchez MD, Olsen PT, Pickett MM, Mangione KJ, et al. Prevalence, distribution, and diversity of Listeria monocytogenes in retail environments, focusing on small establishments and establishments with a history of failed inspections. J Food Prot. 2011;74(7):1083-95. http:// dx.doi.org/10.4315/0362-028X.JFP-10-567 PMID:21740710

21. Endrikat S, Gallagher D, Pouillot R, Hicks Quesenberry H, Labarre D, Schroeder CM, et al. A comparative risk assessment for Listeria monocytogenes in prepackaged versus retail-sliced deli meat. J Food Prot. 2010;73(4):612-9. PMID:20377948

22. Rechel B, Grundy E, Robine JM, Cylus J, Mackenbach JP, Knai C, McKee M. Ageing in the European Union. Lancet 2013;381(9874):1312-22. doi:10.1016/S0140-6736(12)62087-X

23. Broglia A, Kapel C. Changing dietary habits in a changing world: emerging drivers for the transmission of foodborne parasitic zoonoses. Vet Parasitol. 2011;182(1):2-13. http:// dx.doi.org/10.1016/j.vetpar.2011.07.011 PMID:21835548 